\title{
A response to the problem of wild coincidences
}

\author{
Christopher P. Taggart ${ }^{1}[$
}

Received: 16 April 2020 / Accepted: 16 July 2020 / Published online: 27 July 2020

(c) The Author(s) 2020

\begin{abstract}
Derk Pereboom has posed an empirical objection to agent-causal libertarianism: The best empirically confirmed scientific theories feature physical laws predicting no longrun deviations from fixed conditional frequencies that govern events. If agent-causal libertarianism were true, however, then it would be virtually certain, absent 'wild coincidences', that such long-run deviations would occur. So, current empirical evidence makes agent-causal libertarianism unlikely. This paper formulates Pereboom's 'Problem of Wild Coincidences' as a five-step argument and considers two recent responses. Then, it offers a different response: The Problem of Wild Coincidences does not show that current empirical evidence makes agent-causal libertarianism unlikely, even if all events are governed by physical laws featuring fixed long-run conditional frequencies and even if agents can 'overrule' normal physical laws.
\end{abstract}

Keywords Indeterminism $\cdot$ Agency $\cdot$ Conditional probability $\cdot$ Substance-causation

\section{The problem of wild coincidences}

Derk Pereboom endorses hard incompatibilism, according to which 'we likely lack the free will required for moral responsibility since (i) such free will is incompatible with causation by prior events or states, whether deterministic or indeterministic, and (ii) while agent-causation is conceptually coherent', (Baker, pp. 3085-86), it is very unlikely given current empirical evidence. According to agent-causal libertarianism, although our moral responsibility is inconsistent with the causation of our actions entirely by prior events, we are morally responsible for our actions when we, as substances, exercise causal power reasons-responsively ${ }^{1}$ when performing them.

Christopher P. Taggart

c.taggart@surrey.ac.uk

1 University of Surrey, School of Law, Guildford GU2 7XH, UK

1 A substance with causal power that could not exercise it reasons-responsively could not be morally responsible for its behavior. 
Since Pereboom acknowledges that agent-causal libertarianism 'involves no internal incoherence' (Pereboom 1995, p. 28), to 'eliminate' agent-causal libertarianism as a viable alternative to hard incompatibilism he argues that 'we have empirical reasons to believe that it's improbable that we are agent causes as specified by' agent-causal libertarianism. (Pereboom 2014, p. 65). His argument can be called the 'Problem of Wild Coincidences' or the 'Wild Coincidences Objection'. This paper considers two recent responses_-Runyan (2018) and Baker (2017) — and offers a different response. Unlike Runyan's response, this paper's response applies even if all events are governed by physical laws featuring fixed long-run conditional frequencies that never change due to what an agent does from instance to instance. And unlike Baker's response, this paper defends forms of agent-causal libertarianism according to which agent-causes can 'overrule' normal physical laws.

Pereboom's Wild Coincidences Objection: Current empirical evidence ${ }^{2}$ confirms that the physical world, including human brains, is governed by probabilistic laws. Any probabilistic physical law can be analysed as a contingent relation, $R(F, G)$, which holds between two physical ${ }^{3}$ properties, 'F-ness' and 'G-ness'. If $R(F, G)$ obtains, then it entails a fixed long-run conditional frequency: $(x)(\mathrm{P}(\mathrm{G} x \mid \mathrm{F} x)=\mathrm{n})$, where $\mathrm{n} \in[0,1]$. If the probabilistic physical law is deterministic, then $\mathrm{n} \in\{0,1\} .^{4}$ Two clarifications: (1) The bound variable ' $x$ ' in ' $(x)(\mathrm{P}(\mathrm{G} x \mid \mathrm{F} x)=\mathrm{n})$ ' ranges over substances. (2) Assuming an event is a property exemplification by a substance at a time, physical laws predict physical events because physical laws are relations between physical properties exemplified (or not exemplified) by substances at times.

First, assume that all physical laws are deterministic and consider a reasonsresponsive exercise of causal power by an agent performing a token action. When the agent does this:

On the path to action that results from [the agent's] undetermined [exercise of power], alterations in the world, for example in her brain or some other part of her body, are produced. But... at this point... divergences from the deterministic laws [would almost certainly occur]. For the alterations in the world that would result from the undetermined [agential exercises of power] would themselves not be causally determined, and they would thus not be governed by deterministic laws. (Pereboom 2014, p. 66)

Next, assume that some physical laws are not deterministic-some entail $(x)(\mathrm{P}(\mathrm{G} x \mid \mathrm{F} x)=\mathrm{n})$, where $\mathrm{n} \in(0,1)$. Current empirical evidence confirms that, in the long run, physical events are subject to probabilistic laws with fixed conditional frequencies, and the occurrence of physical events is accurately predictable by those fixed frequencies: "[D]espite the existence of libertarian agent-causes who produce

\footnotetext{
2 The 'evidence' is extremely broad. It comprises all observed phenomena that are systematically predictable, in the long run, by hypotheses according to which all physical events are governed by laws entailing probabilistic conditional regularities with specific, fixed frequencies.

${ }^{3}$ Intentional properties are not 'physical' properties because probabilistic physical laws are not 'psychological'. Physical probabilistic laws are not about, for example, 'the probability of antecedent psychological factors (i.e. desires, reasons, inclinations, etc.) leading to given outcomes' (Baker 2017, p. 3092).

${ }^{4}$ By stipulation, a deterministic law is a probabilistic law whose fixed long-run conditional frequency is either 0 or 1 because the law entails either $(x)(\mathrm{F} x \supset \neg \mathrm{G} x)$ or $(x)(\mathrm{F} x \supset \mathrm{G} x)$.
} 
effects in the world... the long-run frequencies of events [are] just as [the laws] would predict' (Pereboom 2001, p. 81).

If reasons-responsive substances causally contributed to the occurrence of physical events in any possible world, then it would be extremely unlikely, for any fixed $\mathrm{n}$, that $(x)(\mathrm{P}(\mathrm{G} x \mid \mathrm{F} x)=\mathrm{n})$ in that world if ' $\mathrm{F}$-ness' and ' $\mathrm{G}$-ness' were involved in human choice and action. The causal activity of reasons-responsive substances is entirely independent of and ungoverned by any probabilistic physical laws governing events. So, it would take 'wild coincidences' for that agent-causal activity, over time, to contribute to bringing about physical events in that world such that $\mathrm{P}(\mathrm{G} x \mid \mathrm{F} x)$ would be fixed and turn out, specifically, to be n:

The proposal that all or even almost all agent-caused free choices should be for just those possible actions the occurrence of whose [physical] components has an extremely high antecedent... probability, and not for any of the alternatives... involves coincidences so wild as to render the proposal incredible. (Pereboom 2001, p. 82)

Pereboom offers an illustration featuring a probabilistic physical law entailing $(x)(\mathrm{P}(\mathrm{G} x \mid \mathrm{F} x)=0.32)$ :

[C]onsider [a] series of possible actions each of which has a physical component whose antecedent probability of occurring is approximately $0.32 \ldots .[\mathrm{I}] \mathrm{t}$ is overwhelmingly likely that for a large number of instances, these components be actually realized close to 32 percent of the time.... If agent-caused free action were compatible with what according to the statistical law is overwhelmingly likely, then for a large enough number of instances, these possible actions would have to be freely chosen close to 32 percent of the time.... But if the occurrence (or non-occurrence) of these physical components is to be settled by the choices of agent-causes, then their actually being chosen close to 32 percent of the time would constitute a coincidence. (Pereboom 2001, pp. 82-83)

Pereboom assumes that the reasons-responsive causal activity of substances making free choices is independent of and unconstrained by the long-run probabilistic laws that govern all physical events. The features of event-causation grounding the fixed long-run conditional frequencies of event-predicting physical laws have no bearing on reasons-responsive exercises of causal power by agents. Pereboom's argument 'conceives of [event-predicting physical] laws as operating in isolation from agentcauses' (Baker 2017, p. 3093). So, it would take wild coincidences for agent-causes to "“fit into" an already complete causal picture' (Baker 2017, p. 3093).

What if, in a possible world, agent-causes generate long-run deviations from the fixed conditional frequency of any 'alleged' physical law entailing $(x)(\mathrm{P}(\mathrm{G} x \mid \mathrm{F} x)=\mathrm{n})$ ? In that world: Over the long run, agent-causes prevent $n-$ or any frequency-from being the fixed conditional frequency of the occurrence of physical events in which substances exemplify 'F-ness' or 'G-ness'. Agent-causes prevent any physical, eventpredicting law from entailing $(x)(\mathrm{P}(\mathrm{G} x \mid \mathrm{F} x)=\mathrm{n})$ for any fixed long-run conditional frequency $\mathrm{n}$. In that world, because of agent-causes, some physical events escape governance by any probabilistic law with a fixed long-run conditional frequency. 
To say that agent-causes can overrule any physical, event-predicting law is to say that agents could exercise causal power such that some event-types would be ungoverned by any physical law with a fixed long-run conditional frequency. Probabilistic physical laws accurately predict all events in the long run in part because agent-causes do not overrule any physical laws over the long run, even though agentcauses could do so. Pereboom argues that it takes extremely unlikely coincidences for agent-causes not to do this even though they could.

To summarize, it is a priori extremely improbable that physical laws predicting all events would feature fixed long-run conditional frequencies if substances independently and reasons-responsively exercised causal power when making free choices. Empirical evidence, however, confirms that all events are governed by probabilistic physical laws featuring fixed long-run conditional frequencies. Therefore, it is empirically improbable that agents perform free actions by exercising undetermined reasons-responsive causal power as substances. Current empirical evidence makes it epistemically unlikely that agent-causal libertarianism is true.

The Problem of Wild Coincidences can be formulated as a five-step argument involving epistemically interpreted conditional probabilities. On this epistemic interpretation, $\mathrm{P}(H \mid E) \in[0,1]$ is high when: If we had good reasons to believe $E$, then we would have good reasons to believe $H . \mathrm{P}(H \mid E) \in[0,1]$ is low when: If we had good reasons to believe $E$, then we would have good reasons to believe $\neg H .^{5}$ Also, $\mathrm{P}(H \mid E)$ $=\frac{\mathrm{P}(H \cap E)}{\mathrm{P}(E)}$, where $\mathrm{P}(E) \neq 0$. Assume that:

$h 1$ is the proposition that all events are governed by physical laws featuring fixed long-run conditional frequencies;

$h 2$ is the proposition that agent-causal libertarianism is true; and

$e$ is the proposition that current empirical evidence confirms that all events are governed by physical laws featuring fixed long-run conditional frequencies.

The Problem of Wild Coincidences ${ }^{6}$ :

(I) Necessarily, if we had good reasons to believe $e$, then we would have good reasons to believe $h 1$

(II) $\mathrm{P}(h 2 \mid h 1)=\frac{\mathrm{P}(h 2 \cap h 1)}{\mathrm{P}(h 1)} \approx 0$

(III) $\mathrm{P}(h 2 \mid e)$ is low [from (I), (II)]

(IV) We have good reasons to believe $e$

$\therefore$ (V) We have good reasons to believe $\neg$ h2 [from (III), (IV)]

Together, premises (I) and (II) very strongly suggest that ${ }^{7}$ (III) If we had good reasons to believe $e$, then we would have good reasons to believe $\neg h 2$. (IV) is the empirical premise that we have good reasons to believe $e$. Therefore, (V) We have

\footnotetext{
5 ' $\mathrm{P}(H \mid E) \in[0,1]$ is low' does not mean: If we had good reasons to believe $E$, then we would have no good reasons to believe $H$.

6 Another version: (I) Necessarily, if we had good reasons to believe $e$, then the epistemic probability of $h 1$ would be high. (II) Epistemically, the probability that $h 1$ and $h 2$ are both true is extremely small. Therefore, (III) Epistemically, $h 2$ would be very unlikely, if we had good reasons to believe $e$. (IV) We have good reasons to believe $e$. Therefore, (V) Epistemically, $h 2$ is very unlikely: We have good reasons to believe $\neg h 2$, that agent-causal libertarianism is false.

7 The inference from (I) and (II) to (III) seems unproblematic as a non-deductive inference.
} 
good (empirical) reasons to believe agent-causal libertarianism is false. '[W]e have empirical reasons to believe that it's improbable that we are agent causes as specified by [agent-causal libertarianism]' (Pereboom 2014, p. 65). ${ }^{8}$

Consider the first premise, (I). (I) is not about the empirical evidence available to us in the actual world. ${ }^{9}$ (I) states that in every possible world in which we have good reasons to believe current empirical evidence confirms that all events are governed by physical laws featuring fixed long-run conditional frequencies, we have good reasons to believe all events are governed by physical laws featuring fixed long-run conditional frequencies. ${ }^{10}$ (I) is an 'analytically' true, a priori non-empirical premise.

Consider the second premise, (II), representing the 'wild coincidences' Pereboom argues it would take for agent-causal libertarianism to be true. (II) states that if we had good reasons to believe all events were governed by physical laws featuring fixed long-run conditional frequencies, then we would have extremely good reasons to believe $h 2$ is false. Assuming agent-causes exercising free will operate independently of event-predicting physical laws, it would take wild coincidences for us to be in a world in which both agent-causal libertarianism is true and all events are governed by physical laws featuring fixed long-run conditional frequencies. If we had good reasons to believe all events were governed by physical laws featuring fixed longrun conditional frequencies, then agent-causal libertarianism would be (extremely!) improbable, epistemically, for us.

\section{Runyan's response}

Jason Runyan points out that Pereboom assumes:

[T] he antecedent probability certain behavior will occur applies in each of a number of occasions and is incapable of changing as a result of what one does from one occasion to the next. There is, however, no evidence this assumption is true. Rather, current [empirical] findings from neuroscience... suggest that the antecedent probability that certain behavior will occur fluctuates from one

\footnotetext{
8 The conclusion of this five-step reconstruction of Pereboom's argument is that we have good empirical reasons to believe agent-causal libertarianism is false, not that we have little empirical reason to believe agent-causal libertarianism is true. This reflects the role the Wild Coincidences Objection plays in Pereboom's defence of hard incompatibilism, which involves ‘four central arguments' (Baker 2017, p. 3086). First, Pereboom argues that 'the kind of free will required for moral responsibility is not based on alternative possibilities, but instead on the... causal history of an action' (Baker 2017, p. 3086; Pereboom 2001, pp. 1-37). Second, Pereboom invokes the 'Disappearing Agent' objection, (Pereboom 2001, pp. 38-50), to argue that an agent's moral responsibility for performing an action requires the agent to be a substance that plays a causal role, as a substance, in the action's aetiology. (At this point, three viable positions remain: agent-causal libertarianism, agent-causal compatibilism, and hard incompatibilism.) Third, Pereboom 'eliminates' agent-causal libertarianism with the Wild Coincidences Objection (Pereboom 2001, pp. 69-88). And fourth, he 'eliminates' compatibilism with the 'Four Case' argument, (Pereboom 2001, pp. 89-125), leaving hard incompatibilism as the remaining viable position. For the Wild Coincidences Objection to 'eliminate' agent-causal libertarianism as a viable position, its conclusion must be that we have good reasons to 'eliminate' that position, to believe agent-causal libertarianism is false.

9 (IV) is.

10 (IV) adds that, in the actual world, we have good reasons to believe current empirical evidence confirms that all events are governed by physical laws featuring fixed long-run conditional frequencies.
} 
occasion to the next and is subject to change depending on what one does.

(Runyan 2018, p. 4564)

Recall that $e$ is the proposition that current empirical evidence confirms that all events are governed by physical laws featuring fixed conditional frequencies, which never fluctuate for any reason, including what an agent does from instance to instance. Runyan describes empirical evidence that some nomological frequencies fluctuate depending on what an agent does from one occasion to the next. This evidence falsifies $e$. Runyan claims there is evidence that there are physical properties involved in human choice and action-say, 'F-ness' and 'G-ness' - such that no physical law entails $(x)(\mathrm{P}(\mathrm{G} x \mid \mathrm{F} x)=\mathrm{n})$ for any fixed $\mathrm{n}$.

We do not have good reasons to believe $e$-(IV) is false-since current empirical evidence confirms that some physical properties involved in human choice and action escape governance by any probabilistic event-predicting physical law featuring a fixed long-run conditional frequency. And, since we lack good reasons to believe $e$, even if $\mathrm{P}(h 2 \mid e)$ is low, we cannot infer that we have good reasons to believe agent-causal libertarianism is false. If (IV) is false, then (V) cannot be inferred confidently, even if (III) is true.

This paper does not question Runyan's argument. If empirical investigation continues to falsify (IV), then the Wild Coincidences Objection remains neutralized. But what if, as more evidence accumulates, we become justified in believing $e$ is true? What if we eventually discover good empirical reasons to believe all events are governed by physical laws featuring fixed long-run conditional frequencies? Runyan's response would falter. An alternative response, unaffected by empirical discovery, might question (II), whether it is true a priori that $\mathrm{P}(h 2 \mid h 1) \approx 0$. Jordan Baker's response does just this for 'integrationist, reconciliatory' agent-causal libertarianism.

\section{Baker's response}

Baker's response to the Wild Coincidences Objection begins with Pereboom's taxonomy of agent-causal views, which includes a term concerning 'how the agent-cause acts in relation to the rest of the world... either the agent-cause follows the normal physical laws of the universe, or... the agent-cause can "overrule" the normal physical laws of the universe' (Baker, p. 3087). When agent-causes cannot overrule normal physical laws, the form of agent-causalism is reconciliatory. As explained, Pereboom's argument targets non-reconciliatory forms of agent-causalism according to which agents can overrule normal physical laws by generating long-run deviations from any fixed conditional frequency, even though agents do not do so.

Following Randolph Clarke, Baker describes an integrated form of reconciliatory agent-causalism that explains why agents cannot overrule normal physical laws. Exercises of substance-causal power by agents making free choices are 'governed along with' event-causation by the event-predicting physical laws:

It [is] (nomologically) impossible, when an agent-causal power is exercised, for the agent alone to cause an event. Causation by appropriate agent-involving events... [is] required in every instance of agent causation.... [W] hen [an] agent 
possessing... agent-causal power acts, it [is] (nomologically) impossible for her action to be caused only by events; it will, as a matter of natural law, be caused by her as well. (Clarke 2003, p. 145)

Under this integrated approach:

[A]gent-causes are best understood as already part of the laws of nature.... [A]gent-causes are already built into the microphysical laws. Thus, there is no space to consider how the action of an agent-cause just so happens to follow the physical probabilities because the agent-cause is already described when considering the probabilities. (Baker 2017, p. 3093)

Baker's response to the Problem of Wild Coincidences defends an integrated, reconciliatory agent-causal view. Pereboom's Problem of Wild Coincidences does not show the following to be improbable: A form of agent-causal libertarianism such that agent-causes cannot overrule physical laws because their integrated causal influence as co-producers of free actions is already 'built into' and 'described' by the fixed long-run conditional frequencies of those laws.

Limiting the target of the Wild Coincidences Objection to integrated, reconciliatory agent-causal libertarianism, its first three steps ${ }^{11}$ become the following, where $h 2^{*}$ is the proposition that integrated, reconciliatory agent-causal libertarianism is true:

(I) Necessarily, if we had good reasons to believe $e$, then we would have good reasons to believe $h 1$

(II)* $\mathrm{P}\left(h 2^{*} \mid h 1\right) \approx 0$

$\therefore$ (III)* $\mathrm{P}\left(h 2^{*} \mid e\right)$ is low [from (I), (II) $\left.{ }^{*}\right]$

Is (II) ${ }^{*}$ true? Is it true that, if we had good reasons to believe $h 1$, then we would have extremely good reasons to believe $h 2^{*}$ was false? Assume we had good reasons to believe $h 1$. Under integrated, reconciliatory agent-causalism, no long-run deviations from the fixed frequencies featured in the physical laws governing all events occur. So, integrated, reconciliatory agent-causalism would fit seamlessly with what we had good reasons to believe - that all events were governed by physical laws featuring fixed long-run conditional frequencies. We would not know whether integrated, reconciliatory agent-causalism is true or agent-causalism is false: '[W]ithin an integrationist framework, empirical considerations alone [could not] provide evidence against a reconciliatory agent-cause' (Baker 2017, p. 3094). Thus, our having good reasons to believe $h 1$ would not give us extremely good reasons to believe integrated, reconciliatory agent-causal libertarianism is false, i.e., $\neg\left(\mathrm{P}\left(h 2^{*} \mid h 1\right) \approx 0\right)$.

Limiting the target of Pereboom's objection to integrated, reconciliatory agentcausal libertarianism, (II) ${ }^{*}$ is false. If (II) ${ }^{*}$ is false, then (III) ${ }^{*}$ cannot be inferred confidently, even though (I) is true. And without (III) ${ }^{*}$, even if (IV) is true, we cannot confidently infer $(\mathrm{V})^{*}$, that we have good reasons to believe $\neg h 2^{*}$ - that integrated, reconciliatory agent-causal libertarianism is false.

This paper does not question Baker's argument. If we limit the discussion to integrated, reconciliatory forms of agent-causal libertarianism, then, as explained,

\footnotetext{
11 (IV) remains the same, and the last step of the modified objection becomes: (V) We have good reasons to believe $\neg h 2^{*}$.
} 
$\neg\left(\mathrm{P}\left(h 2^{*} \mid h 1\right) \approx 0\right)$. No longer must 'wild coincidences' be occurring for long-term deviations from the fixed frequencies of event-predicting, physical laws not to occur. Integrated, reconciliatory agent-causal libertarianism is safe from the Problem of Wild Coincidences.

Unlike Baker's response, the next section's response to the Wild Coincidences Objection grants Pereboom's assumption that the reasons-responsive causal activity of substances making free choices is independent of and unconstrained by the longrun probabilistic physical laws governing all events. It accepts that agent-causes do not overrule normal physical laws even though they can. It accepts that physical laws accurately predicting events in the long run have the fixed conditional frequencies they do because of the nature of event-causation only and that the causal influences of agents over time could interfere with this but do not.

\section{A different response}

This section's response to the Problem of Wild Coincidences lifts Sect. 3's restriction to integrated, reconciliatory agent-causal libertarianism. For the sake of argument, it grants Pereboom's assumptions that: (i) The reasons-responsive causal activity of substances making free choices is entirely independent of the long-run probabilistic physical laws that govern all events; and (ii) Agent-causes do not overrule normal physical laws even though they can. Recall the Wild Coincidences Objection:

(I) Necessarily, if we had good reasons to believe $e$, then we would have good reasons to believe $h 1$

(II) $\mathrm{P}(h 2 \mid h 1)=\frac{\mathrm{P}(h 2 \cap h 1)}{\mathrm{P}(h 1)} \approx 0$

(III) $\mathrm{P}(h 2 \mid e)$ is low [from (I), (II)]

(IV) We have good reasons to believe $e$

$\therefore$ (V) We have good reasons to believe $\neg$ h2 [from (III), (IV)]

What a priori reasons are there to believe that (II), $\mathrm{P}(h 2 \mid h 1)=\frac{\mathrm{P}(h 2 \cap h 1)}{\mathrm{P}(h 1)} \approx 0$, is true once Sect. 3's restriction is lifted? (II)'s extremely small value assignment to $\mathrm{P}(h 2 \mid h 1)$ represents the idea that it would take 'wild coincidences' for the longrun exercise of reasons-responsive substance-causal power-ungoverned by physical, event-predicting laws - not to overrule those laws by generating long-run deviations from the laws' fixed conditional frequencies. (II) is not an empirical premise. Any reason we have to believe (II) is independent of any empirical evidence $e$ refers to. Do we have any such ' $e$-independent' a priori reasons to believe $\frac{\mathrm{P}(h 2 \cap h 1)}{\mathrm{P}(h 1)} \approx 0$ ? If we have no such a priori reasons, then the Problem of Wild Coincidences fails as an empirical objection to agent-causal libertarianism.

Consider a probability space: $(\Omega, \Sigma, \mathrm{P}) . \Omega$ is the collection of all possible worlds, interpreted as maximal states of affairs. $\Sigma$ is a $\sigma$-algebra on $\Omega$ containing all and only subsets of $\Omega$ measurable by P. Subsets of $\Omega$ are sets of possible worlds, and sets of possible worlds are interpreted as states of affairs, or propositions. So interpreted, $\Sigma$ is the collection of all propositions measurable by $\mathrm{P}$, including 'the necessarily true proposition', $\Omega$, and 'the necessarily false proposition', $\emptyset$. P is the probability space's measure, a function assigning each measurable proposition in $\Sigma$ a probability, $\mathrm{n} \in$ 
$[0,1]$. ' $\mathrm{P}(\Omega)=1$ ' represents an epistemic probability of 100 percent that the actual world, $\alpha$, is a possible world; ' $\mathrm{P}(\emptyset)=0$ ' represents an epistemic probability of zero percent that $\alpha$ is not a possible world; ' $\mathrm{P}(h 2 \mid h 1)=j \in[0,1]$ ' represents an epistemic probability of $j$ that $\alpha$ entails $(h 1 \& h 2)$; ' $\mathrm{P}(h 1)=k \in[0,1]$ ' represents an epistemic probability of $k$ that $\alpha$ entails $h 1$. Diagrammatically:

Fig. $1 j \in[0,1]$ is the epistemic probability that $\alpha$ is in the shaded area

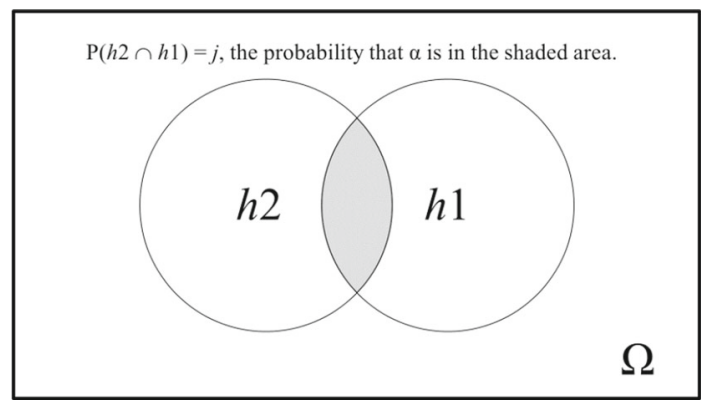

Fig. $2 k \in[0,1]$ is the epistemic probability that $\alpha$ is in the shaded area
$\mathrm{P}(h 1)=k$, the probability that $\alpha$ is in the shaded area.

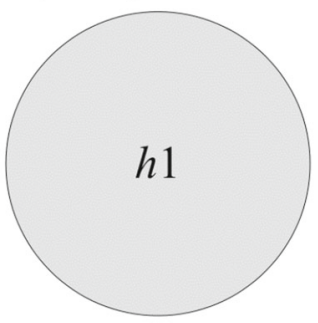

$\mathrm{P}(h 2 \mid h 1)=\frac{\mathrm{P}(h 2 \cap h 1)}{\mathrm{P}(h 1)} \approx 0$ if and only if $\frac{j}{k} \approx 0$. Since $k \leq 1, \frac{j}{k} \approx 0$ only if $j$ is extremely small. So, the Problem of Wild Coincidences provides us no good empirical reasons to believe agent-causal libertarianism is false unless we have good a priori reasons to believe $j$ is extremely small. $j$ is extremely small if and only if: (i) ( $h 2 \cap h 1)$ is measurable by $\mathrm{P}$ and (ii) $\mathrm{P}(h 2 \cap h 1) \approx 0$. Therefore, we have good a priori reasons to believe $j$ is extremely small only if we have good a priori reasons to believe (i) and good a priori reasons to believe (ii).

Do we have good, $e$-independent a priori reasons to believe $(h 2 \cap h 1)$ is measurable by $\mathrm{P}$ ? We have no grounds to doubt the Axiom of Choice or that $(\Omega, \Sigma, \mathrm{P})$ is isomorphic to a Lebesgue measure space or another type of space with non-measurable subsets. (If $(\Omega, \Sigma, \mathrm{P})$ is isomorphic to a Lebesgue measure space, then there are as many nonmeasurable propositions as measurable ones.) ${ }^{12}$ What $e$-independent a priori reason do we have to believe $(h 2 \cap h 1)$ is measurable by P? Whether or not $\mathrm{P}$ is a Lebesgue measure, $\mathrm{P}$ could be defined as assigning a value $\mathrm{n} \in[0,1]$ to $(h 2 \cap h 1)$ by fiat. But

\footnotetext{
12 I thank an anonymous reviewer for identifying and clarifying these technical points.
} 
what non-arbitrary, $e$-independent a priori reasons could motivate such a definitional stipulation? What value $\mathrm{n}$ is to be assigned? And what non-arbitrary, $e$-independent a priori reasons motivate assigning that value to $(h 2 \cap h 1)$ ?

In the absence of any non-arbitrary, $e$-independent a priori reasons to assign any value $\mathrm{n} \in[0,1]$ to $(h 2 \cap h 1)$, we lack good a priori reasons to believe $(h 2 \cap h 1)$ is measurable by P. We have good a priori reasons to believe $j$ is extremely small only if we have good a priori reasons to believe $(h 2 \cap h 1)$ is measurable by P. The Problem of Wild Coincidences gives us good empirical reasons to disbelieve agent-causal libertarianism only if we have good a priori reasons to believe $j$ is extremely small. Therefore, the Problem of Wild Coincidences does not give us good empirical reasons to disbelieve agent-causal libertarianism unless $w e$ have non-arbitrary, $e$-independent a priori reasons to assign a value $\mathrm{n} \in[0,1]$ to $(h 2 \cap h 1)$. And for the Problem of Wild Coincidences to give $u s$ good empirical reasons to disbelieve agent-causal libertarianism, we must have $e$-independent a priori reasons to assign an extremely small value to $(h 2 \cap h 1)$.

Imagine that Perry is a hard incompatibilist who endorses the Problem of Wild Coincidences. Does Perry have any $e$-independent a priori reasons to believe $j=\mathrm{P}(h 2 \cap h 1)$ is extremely small? In possible worlds in the shaded area in Fig. 1, agent-causal libertarianism is true and there are no long-run deviations from eventpredicting physical laws. Not all worlds in Fig. 1's shaded area have $\alpha$ 's physical laws. But, whatever long-run event-predicting probabilistic physical laws those worlds have, libertarian agent-causes in those worlds can overrule those laws even though they do not. Two 'candidate' $e$-independent reasons to believe $j=\mathrm{P}(h 2 \cap h 1)$ is extremely small will now be considered.

First Candidate Reason: Consider two transfinite sets. S1 contains every state of affairs in which libertarian agent-causes capable of doing so generate long-run 'deviations' from some 'alleged' event-predicting probabilistic physical laws. In all states of affairs in S1, $h 2$ is true, but $h 1$ is false-some physical events escape governance by laws featuring fixed long-run conditional frequencies. S2 contains every state of affairs in which libertarian agent-causes capable of doing so do not generate long-run 'deviations' from event-predicting physical laws, and all physical events are governed by such laws. In all states of affairs in S2, both $h 1$ and $h 2$ are true.

Perry's first 'candidate' $e$-independent reason to believe $j$ is extremely small is that S1 seems much 'larger' to him than S2. A priori, there seem to be many more possible ways for libertarian agent-causes capable of generating long-run deviations to generate such deviations than possible ways for them not to. When Perry focuses on one lengthy possible state of affairs in which no long-run deviations occur despite the ongoing independent causal activity of agents performing free actions, an arbitrarily large number of equally lengthy possible states of affairs in which those free agents overrule at least one physical law can be 'brought to mind'. He thereby intuits, a priori, that $j$ is extremely small.

Now imagine Libby, an agent-causal libertarian who disagrees with Perry about (II). She replaces (II) with (II)' and argues as follows that agent-causal libertarianism is epistemically very likely for us: 
(I) Necessarily, if we had good reasons to believe $e$, then we would have good reasons to believe $h 1$

(II) $)^{\prime} \mathrm{P}(h 2 \mid h 1)=\frac{\mathrm{P}(h 2 \cap h 1)}{\mathrm{P}(h 1)} \approx 1$

(III) $^{\prime} \mathrm{P}(h 2 \mid e)$ is high [from (I), (II) $\left.{ }^{\prime}\right]$

(IV) We have good reasons to believe $e$

$\therefore(\mathrm{V})^{\prime}$ We have good reasons to believe $h 2$ [from (III) $\left.{ }^{\prime},(\mathrm{IV})\right]$

To apply an analysis to (II) ${ }^{\prime}$ similar to the preceding analysis of (II): $\mathrm{P}(h 2 \mid h 1)=$ $\frac{\mathrm{P}(h 2 \cap h 1)}{\mathrm{P}(h 1)} \approx 1$ if and only if $\frac{j}{k} \approx 1$, where $j$ is the likelihood that $\alpha$ is in the shaded area in Fig. 1, and $k$ is the likelihood that $\alpha$ is in the shaded area in Fig. 2. The more reason we have to believe $j \approx k$, the more reason we have to believe $\frac{j}{k} \approx 1$ and (II) ${ }^{\prime}$ is true. The less reason we have to believe $j \approx k$, the less reason we have to believe $\frac{j}{k} \approx 1$ and (II) ${ }^{\prime}$ is true. Libby's argument gives us good empirical reasons to believe agent-causal libertarianism only if we have good a priori reasons to believe $j \approx k$.

Imagine that Libby believes $j \approx k$ because S2 seems much 'larger' to her than $\mathrm{S} 1$. A priori, there seem to be many more possible ways for libertarian agent-causes capable of generating long-run deviations not to generate such deviations than possible ways for them to do so. When Libby focuses on one lengthy possible state of affairs in which long-run deviations occur due to the ongoing independent causal activity of agents performing free actions, an arbitrarily large number of equally lengthy possible states of affairs in which those free agents never overrule any physical laws can be 'brought to mind'. She thereby intuits, a priori, that $j \approx k$.

From an a priori 'point of view from eternity', Perry's intuition and Libby's intuition are on a par with each other. Each intuition is undergirded by a similar a priori reflection on the 'conceptual space' of all possible states of affairs. Their reflections symmetrically 'oppose' one another and, when 'fed into' the same five-step 'argument form' at the second step, each generates the opposite conclusion about what we have good empirical reasons to believe about agent-causal libertarianism. Consider Fig. 3:

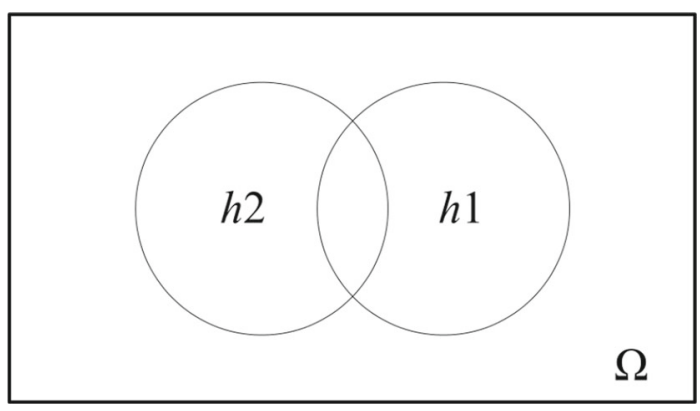

Fig. 3 This figure divides $(h 2 \cup h 1)$ into three regions from left to right: $\left(h 2 \cap h 1^{c}\right),(h 2 \cap h 1)$, and $\left(h 2^{c} \cap\right.$ $h 1)$ 
Perry's intuition convinces him that $\mathrm{P}(h 2 \cap h 1) \approx 0$, and Libby's intuition convinces her that $\mathrm{P}\left(h 2^{c} \cap h 1\right) \approx 0$. But what intuitive, $e$-independent a priori basis (not begging any questions) could give $u s$ any more reason to believe $\mathrm{P}(h 2 \cap h 1) \approx 0$ than to believe $\mathrm{P}\left(h 2^{c} \cap h 1\right) \approx 0$ ? No such basis exists, unless we can accurately, reliably, and directly intuit, a priori, the 'sizes' (in the 'probability measure' sense) of transfinite subsets of possible worlds in $\Omega$. We have no such ability. None of this shows Perry's belief that $j$ is extremely small or Libby's belief that $j \approx k$ is false. But it does show Perry has no good $e$-independent a priori reasons to believe $j$ is extremely small, any more than Libby has any good $e$-independent a priori reasons to believe $j$ $\approx k$.

Second Candidate Reason: Perry might have a different $e$-independent reason ${ }^{13}$ to believe $j$ is extremely small consisting of part of his reasoning. Perhaps Perry is making an $e$-independent abductive inference, which is $e$-independent in that it is available in any possible world in which $h 1$ is true and empirical evidence (not necessarily the same as the empirical evidence available to $u s$ in the actual world) confirms $h 1$. Imagine a possible world, $W \neq \alpha$, in which: (i) Carey exists; (ii) $h 1$ is true; and (iii) Carey has good empirical evidence (different from the empirical evidence available to us in $\alpha$ ) that $h 1$ is true. In $W$, Carey argues that agent-causal libertarianism is probably false (in $W$ ) as follows:

There must be some explanation why $h 1$ is true (in $W$ ). Whatever that explanation is, call it ' $\varepsilon$ '. Does $\varepsilon$ entail $\neg h 2$ or not ${ }^{14}$ It would be simpler if $\varepsilon$ entailed $\neg h 2$. If $h 2$ were true (in $W$ ), then I would need to explain why agent-causes do not overrule event-predicting physical laws (in $W$ ) even though they could. I can't see what that explanation might be. If $\neg h 2$, then there would be less to explain. The empirical evidence I have (in $W$ ) gives me good reasons to believe $h 1$ (in $W$ ). And having good reasons to believe $h 1$ (in $W$ ), combined with an abductive inference to the best explanation of $h 1$, gives me good reasons to believe $\varepsilon$, the simplest explanation of $h 1$, is true (in $W$ ). Whatever $\varepsilon$ 's details are, for $\varepsilon$ to be the simplest explanation of $h 1, \varepsilon$ must at least entail $\neg h 2$. So, my abductive explanatory reason to believe $\varepsilon$ (in $W$ ) is also a good reason to believe $\neg h 2$ (in $W$ ). In sum: Given the empirical evidence I have (in $W$ ) that all events are governed by physical laws featuring fixed long-run conditional frequencies, I have good reasons to believe agent-causal libertarianism is false (in $W$ ). I conclude that agent-causal libertarianism is probably false (in $W)$.

\footnotetext{
13 Arguably, this 'candidate' reason is not an a priori reason. But it is, in a sense, $e$-independent, and considering it is illuminating.

14 There are two ways $\varepsilon$ might not entail $\neg h 2$ : $\varepsilon$ entails $h 2$ or $\varepsilon$ entails neither $h 2$ nor $\neg h 2$.
} 
Where in the foregoing is any reason for Carey to believe $j$ is extremely small and (II) is true? Consider Fig. 4:

Fig. $4 j \in[0,1]$ is the epistemic probability that $W$ is in the shaded area

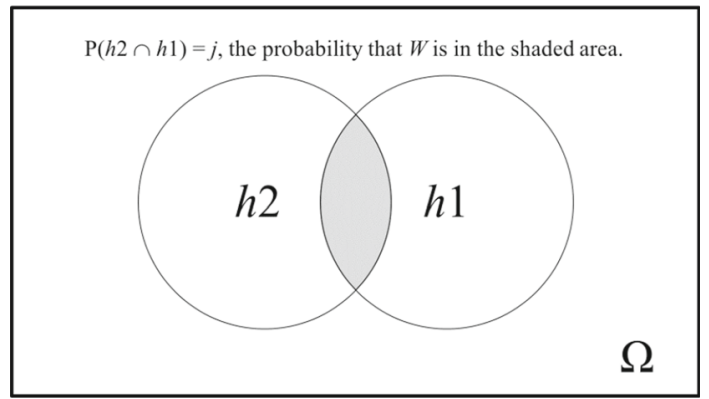

Carey must believe the evidence he has that $h 1$ is true in $W$ gives him an extremely good reason to believe he is in a possible world, $W$, located inside the $h 1$ circle and outside the shaded area in Fig. 4. Once he believes he is there, he believes agent-causal libertarianism is false in $W$ because his possible world is in the complement of the $h 2$ circle. How does Carey get from having good reasons to believe he is in the $h 1$ circle to being confident he is not in the shaded area in Fig. 4? There seems to be only one way-by assuming that, relative to $\Omega$, the density of possible worlds in the shaded area in Fig. 4 is extremely small and that $j$ is extremely small. Does this mean that the second 'candidate' $e$-independent reason to believe $j$ is extremely small, a reason embodied in Carey's abductive inference on the way to concluding he has good reasons to believe $\neg h 2$, is the assumption that $j$ is extremely small? Something must have gone wrong. The second 'candidate' $e$-independent reason to believe $j$ is extremely small is supposed to be an abductive inference, not the assumption that $j$ is extremely small.

Reconsider the abductive inference that Carey (and, perhaps, Perry) make on their way to concluding they have good reasons to believe agent-causal libertarianism is false in their worlds. Their empirical evidence confirms $h 1$ is true. There must be an explanation, $\varepsilon$, of $h 1 .^{15}$ In one sense, the explanation of $h 1$ would be 'simpler' if $\varepsilon$ entailed $\neg h 2$. Perhaps Carey and Perry have good reasons to believe $\neg h 2$ if an explanation's simplicity is a good reason to believe it and anything it entails. ${ }^{16}$ Perhaps what gets Carey and Perry from being confident they are in the $h 1$ circle to being confident they are in the complement of the $h 2$ circle is the simplicity of $\varepsilon$, a simplicity that $\varepsilon$ has only if $\varepsilon$ entails $\neg h 2$.

Assume $\varepsilon$ 's simplicity as an explanation of $h 1$ is a good reason to believe that $\varepsilon$, and therefore any proposition $\varepsilon$ entails, is true. If $\varepsilon$ explained $h 1$ without entailing $h 2$, then $\varepsilon$ would be a simpler explanation of $h 1$ than if $\varepsilon$ explained $h 1$ just as well, but only by entailing $h 2$. An explanation of $h 1$ making no reference to agent-causal libertarianism is simpler than one explaining $h 1$ equally well, but only by positing agent-causal libertarianism. So, Carey and Perry have good reasons to believe $\varepsilon$, the

\footnotetext{
15 Who knows what $\varepsilon$ is? For simplicity, ignore the possibility that the explanation of $h 1$ in $\alpha$ might differ from the explanation of $h 1$ in $W$.

16 This paper is neutral about whether an explanation's simplicity is a good reason to believe the explanation, including anything it entails.
} 
simplest explanation of $h 1$, does not entail $h 2$. But Carey and Perry do not have good reasons to believe $\varepsilon$ entails $\neg h 2$. Contrast the following:

(A) $\varepsilon$ would be simpler if $\varepsilon$ entailed $\neg h 2$ than if $\varepsilon$ entailed $h 2$.

(B) $\varepsilon$ would be simpler if $\varepsilon$ did not entail $h 2$ than if $\varepsilon$ entailed $\mathrm{h} 2$.

Consider (A). Explanatory matters would be simpler if $\varepsilon$ entailed $\neg h 2$ than if $\varepsilon$ entailed $h 2$. They would be simpler because, when Carey and Perry set out to explain $h 1$, if they acquired good reasons to believe $\varepsilon$ entailed $\neg h 2$, then, in addition to finding the simplest explanation of $h 1$, they would receive a collateral benefit-they would never need to explain why agent-causes that can overrule event-predicting physical laws never do. But Carey and Perry receive that benefit only if $\varepsilon$ 's simplicity as an explanation of $h 1$ turns on eliminating, as opposed to ignoring, agent-causal libertarianism.

Their abductive inference to $\varepsilon$ ignores reasons-responsive substance causation unless and until it cannot. Until they need reasons-responsive substance causation to explain why all events are governed by physical laws featuring fixed long-run conditional frequencies, their explanation of $h 1$ says nothing about agent-causal libertarianism. And there is no reason to believe they must posit reasons-responsive substance causation to explain $h 1$. Their need to explain $h 1$ gives them no reason to believe in libertarian agent-causes. Whether they, or we, might have other reasons to believe in them is another matter.

Before the heliocentric theory, Ptolemaic epicycles were needed to explain the motions of the moon, Sun, and planets. So, before the heliocentric theory, we had good reasons to believe in forces generating Ptolemaic epicycles. After the heliocentric theory, which explained the phenomena more simply, we lost our empirical reasons to believe in Ptolemaic epicycle-forces. Did losing those reasons amount to a reason to believe there were no Ptolemaic epicycle-forces? Yes and no. We now disbelieve in Ptolemaic epicycle-forces, in part, because we have no reason, empirical or otherwise, to believe in them.

Imagine that philosophical argumentation convinced us that libertarian free will was possible only if there were Ptolemaic epicycle-forces. Further, imagine that additional philosophical argumentation convinced us that libertarian free will was necessary to justify reactive attitudes and practices, such as resentment and legal punishment. And imagine that we were convinced that such reactive attitudes and practices could be justified. In such a case, losing our empirical reasons to believe in Ptolemaic epicycleforces would not be a reason for us to believe there were no Ptolemaic epicycle-forces.

To recapitulate: Carey and Perry have strong empirical evidence they are in the $h 1$ circle. To cast doubt upon agent-causal libertarianism, they must find a good reason to believe they are in the complement of the $h 2$ circle. Their abductive inference to $\varepsilon$, the simplest explanation of $h 1$, gives them a good reason to believe they are in the complement of the $h 2$ circle only if $\varepsilon$ 's simplicity requires that $\varepsilon$ entail $\neg h 2$. But $\varepsilon$ 's simplicity requires only that $\varepsilon$ not entail $h 2$. Therefore, their abductive inference does not give Carey and Perry a good reason to believe they are in the complement of the $h 2$ circle. They lack any empirical reason to believe agent-causal libertarianism is false.

As previously explained, the Problem of Wild Coincidences gives us empirical reasons to disbelieve agent-causal libertarianism only if we have good a priori reasons 
to believe $j$ is extremely small. Pereboom's objection tries to convince us we are in the complement of the $h 2$ circle by convincing us we are in the $h 1$ circle but not in the shaded area in Fig. 1. Because Carey and Perry are not relying on the first 'candidate' reason, they are left without any reason to believe $j$ is extremely small and (II) is true. They must assume $j$ is extremely small to try to convince us that they, and we, are in possible worlds in which agent-causal libertarianism is probably false.

The Problem of Wild Coincidences gives us empirical reasons to disbelieve agentcausal libertarianism only if we have good $e$-independent a priori reasons to believe $j$ is extremely small. We have such reasons only if we have good $e$-independent a priori reasons to believe both: (i) $(h 2 \cap h 1)$ is measurable by $\mathrm{P}$ and (ii) $\mathrm{P}(h 2 \cap h 1) \approx 0$. But we lack any such reasons. So, the Problem of Wild Coincidences does not show that current empirical evidence makes agent-causal libertarianism unlikely. And it does not show this even if all events are governed by physical laws featuring fixed long-run conditional frequencies and agents can overrule those laws, even though they never do.

Acknowledgements I wish to thank Stephen Bero, Bebhinn Donnelly-Lazarov, Connor Healy, Ambrose Lee, Alex Sarch, and anonymous referees for helpful comments on earlier drafts.

Open Access This article is licensed under a Creative Commons Attribution 4.0 International License, which permits use, sharing, adaptation, distribution and reproduction in any medium or format, as long as you give appropriate credit to the original author(s) and the source, provide a link to the Creative Commons licence, and indicate if changes were made. The images or other third party material in this article are included in the article's Creative Commons licence, unless indicated otherwise in a credit line to the material. If material is not included in the article's Creative Commons licence and your intended use is not permitted by statutory regulation or exceeds the permitted use, you will need to obtain permission directly from the copyright holder. To view a copy of this licence, visit http://creativecommons.org/licenses/by/4.0/.

\section{References}

Baker, Jordan. (2017). Rejecting Pereboom's empirical objection to agent-causation. Synthese, 194, 3085-3100.

Clarke, Randolph. (2003). Libertarian accounts of free will. Oxford: Oxford Univ. Press.

Pereboom, Derk. (1995). Determinism Al Dente. Noûs, 29, 21-45.

Pereboom, D. (2001). Living without free will. Cambridge: Cambridge Univ. Press.

Pereboom, D. (2014). Free will, agency, and meaning in life. Oxford: Oxford Univ. Press.

Runyan, Jason D. (2018). Agent-causal libertarianism, statistical neural laws and wild coincidences. Synthese, 195, 4563-4580.

Publisher's Note Springer Nature remains neutral with regard to jurisdictional claims in published maps and institutional affiliations. 